\title{
Suppression of Drill-String Stick-Slip Vibration
}

\author{
Vahid Vaziri $^{1, \star}$, Marcin Kapitaniak ${ }^{1, \star \star}$, and Marian Wiercigroch ${ }^{1, \star \star \star}$ \\ ${ }^{1}$ Centre for Applied Dynamic Research, University of Aberdeen, UK
}

\begin{abstract}
We investigate experimentally and numerically suppression of drill-string torsional vibration while drilling by using a sliding mode control. The experiments are conducted on the novel experimental drilling rig developed at the University of Aberdeen [1] and using PDC commercial drill-bits and real rock-samples. A mathematical model of the experimental setup which takes into account the dynamics of the drill-string and the driving motor, is proposed. Then a sliding mode control method is employed to suppress stick-slip oscillations. The experimental and numerical results considering a time delay of the actuator are in a close agreement. Stick-slip vibration is eliminated and significant reduction in vibration amplitude has been observed when using the controller.
\end{abstract}

\section{Introduction}

During a downhole drilling process excessive vibrations can occur, which in most cases have a negative impact on the effectiveness of the process and the drilling equipment [2]. Such vibration may lead to an accelerated wear and premature damage of the expensive drilling equipment. Often different dynamic effects such as bit-bounce, stick-slip, forward and backward whirls may appear in the drilling process. Recently several attempts have been made to replicate these vibrations in academic laboratories. However, in most studies the cutting process is simulated by a friction between two disks [3].

In this work we focus on suppression of stick-slip phenomenon while drilling, which nature is still not well understood and can lead to a catastrophic failure of the drill-stings. Therefore, firstly, a 2-DOF lumped mass model is developed using the torque-on-bit curves, which capture both the frictional and cutting compo-

\footnotetext{
^e-mail: vahid.vaziri@abdn.ac.uk

$\star \star$ e-mail: marcin.kapitaniak@abdn.ac.uk

$\star \star \star$ e-mail: m.wiercigroch@abdn.ac.uk
}

nents of the drill-bit rock interactions [4]. Then, a sliding surface is defined and consequently a sliding mode controller is developed which accommodates the parameters uncertainties. The experimental and numerical results demonstrate the predictive capabilities of the mathematical models and the power of the controller to eliminate the stick-slip vibrations in the given conditions.

\section{Drill-String Experimental Stand}

A novel experimental drilling facility has been designed and built at the University of Aberdeen, capable of reproducing all major types of drill-string vibration [4-7]. It allows to investigate nonlinear behaviour between the drillbit and the formation and to introduce different control methods of vibration suppression. Unlike typical other research experimental academic facilities, our rig is configured in such a way that the cutting process is undertaken us- 
ing real commercial drill-bits and rock samples. The rig shown in Fig. 1 is equipped with a variety of different sensors and transducers. Angular positions are measured by two quadrature encoders having 500 pulse per revolution, where axial motion of the drill-bit is captured by a P1010 position transducer attached to the Bottom Hole Assembly. Horizontal and vertical forces as well as torque coming from the bit to the rock are measured by a 4-component Kistler dynamometer placed under the rock sample. All the voltage signals are sent to a NI PCIe-6321 data acquisition card which has multiple analogue input (16-Bit, $250 \mathrm{kS} / \mathrm{s}$ ) and output (900 $\mathrm{kS} / \mathrm{s}$ ) channels and four 32 bit counters/timers. Accessing to 4 counters, allows us to precisely synchronise two encoders. This is important as then the drill-pipe twist can be calculated accurately. Furthermore, the card high performance allows high frequency data sampling up to 30 $\mathrm{kHz}$. This card is controlled by a LabVIEW programme with custom built graphical interface allowing to monitor responses of the system and to present time histories of variables and phase portraits in real-time. This program also sends the command to the top AC motor through the NI card and the ABB frequency convertor. This convertor can work in speed or torque control modes which control the velocity or torque provided by the motor. Different speed or torque nonlinear control methods can be implemented in the LabVIEW program such as a sliding mode control in Fig.1.

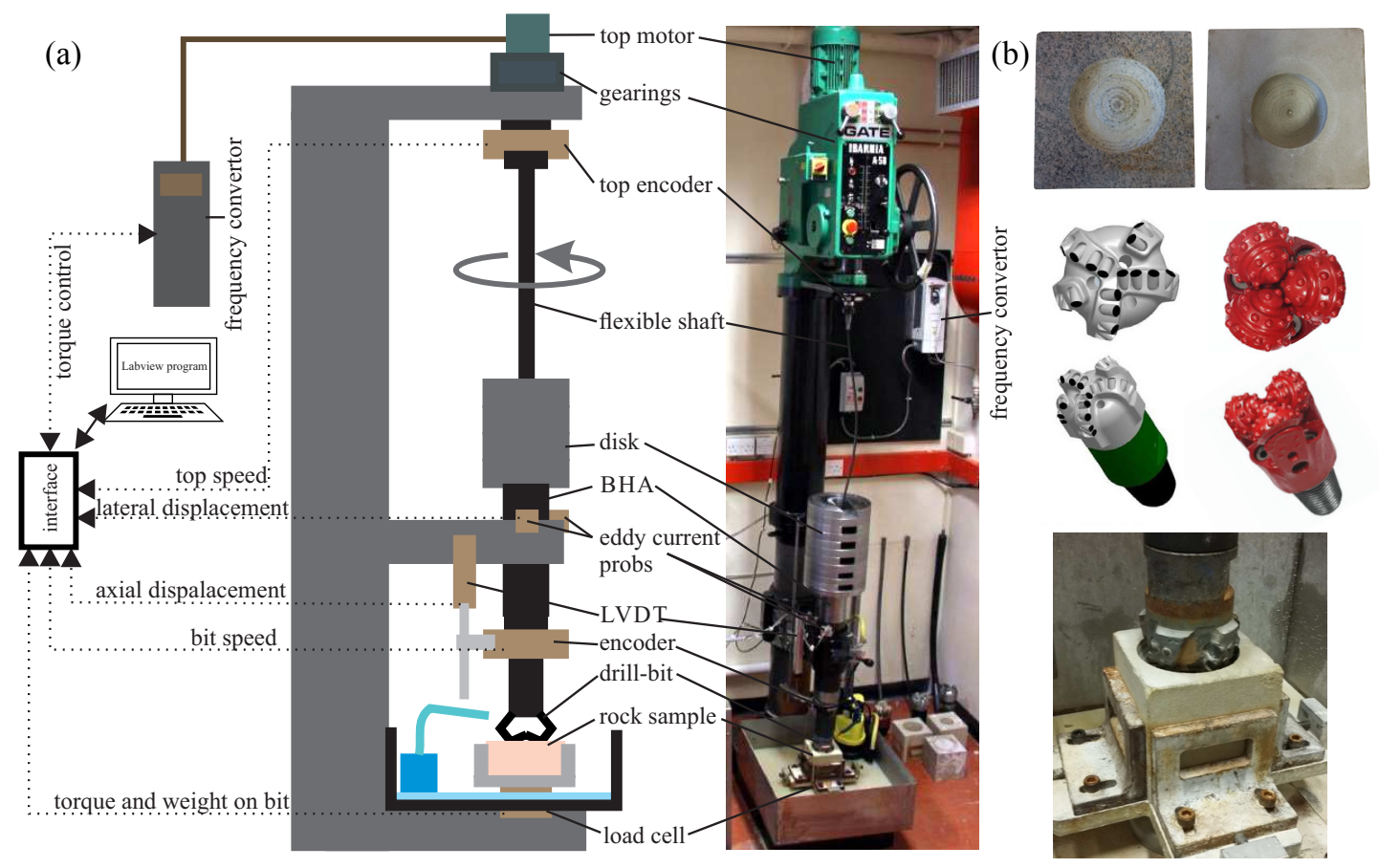

Figure 1. (a) Schematic diagram of the experimental setup, (middle) photograph of the experimental rig and (b) examples of rock samples and drill-bits used in the setup. Main components of the system are: sensors (top and bottom encoders, eddy current probes, LVDT and 4-component load cell), electric motor, flexible or rigid shafts, disks, the BHA, drill-bit and rock samples. 
(a)
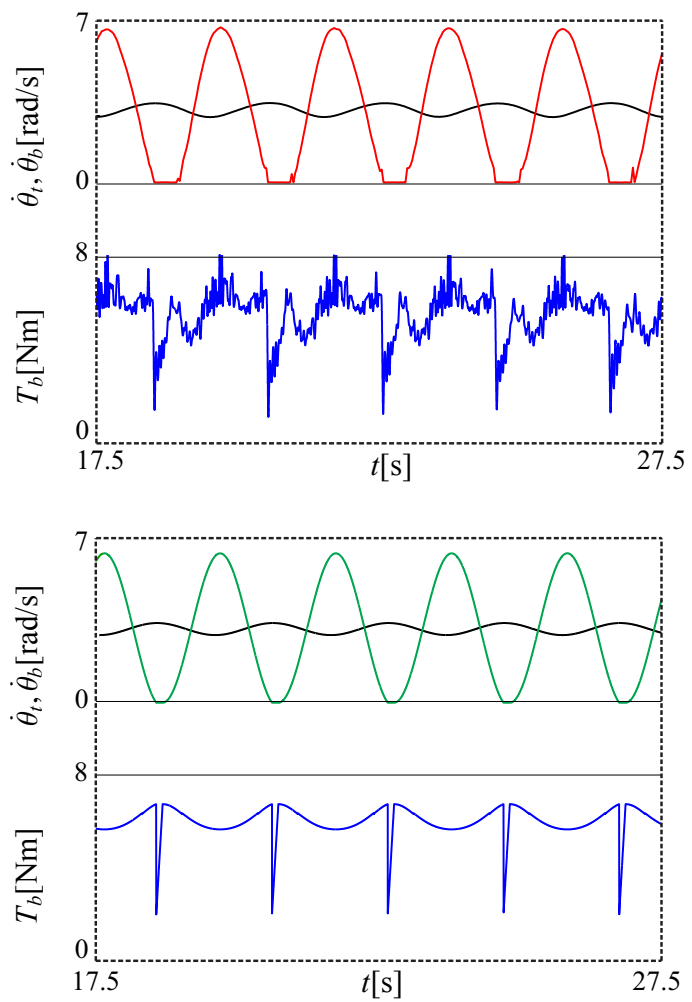

(b)

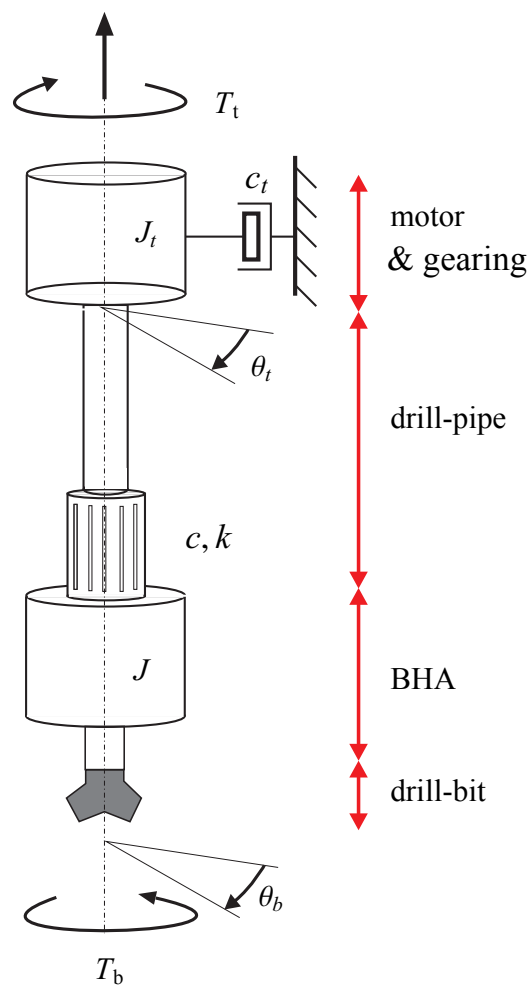

Figure 2. (a) An example of stick-slip oscillations occurring in the experimental rig for $W_{b}=1.79 \mathrm{kN}$ and 1.5 inch pre-buckled flexible shaft. The time histories of the angular velocities at the bit, $\dot{\theta}_{b}$, and the surface, $\dot{\theta}_{t}$ together with TOB obtained in experimental studies (top) and calculated using proposed model (bottom). (b) A physical model of a 2-DOF lump mass torsional system. The viscous damping property of the motor and gearing system and the visco-elasto properties of the pipe are given by $c_{t}, c$ and $k$, respectively. The reactive torque acting on the system during drilling is represented by $T_{b}$.

\section{Mathematical Model and Sliding Model Control}

The most used class of models for capturing uncoupled torsional vibration consists of several parallel disks, rotating around their common axis and connected to each other by torsional spring and damper. Top disk in all these models represents the rotary table and the bottom disk represents the drill-bit. The bit-rock interaction is modeled by the velocity dependent resistive torque acting on the bottom disk. This class of models has been widely employed in studies focusing in drill-string torsional vibra- tion. The number of disks varies in those studies, for example 2 disks [8], 3 disks [9], 4 disks [10] and even 18 disks [11]. In the current work a 2-disk model has been employed (see Fig.2(b)) to model the CADR experimental drilling rig, following the work presented in [8]. The viscous damping property of the motor and gearing system and the visco-elasto properties of the drill-pipe are given by $c_{t}, c$ and $k$, respectively. The reactive torque acting on the system during drilling is represented by $T_{b}$. In this model, the frequency convertor is set in the torque control mode and the top velocity can be calculated from the equation of motion. 
(a)

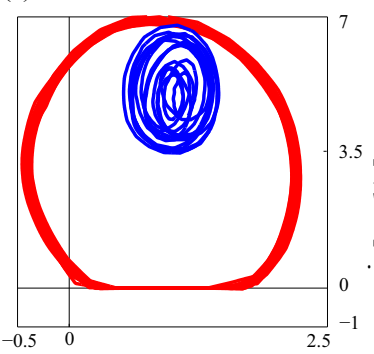

$\theta_{t}-\theta_{b}[\mathrm{rad}]$

(d)

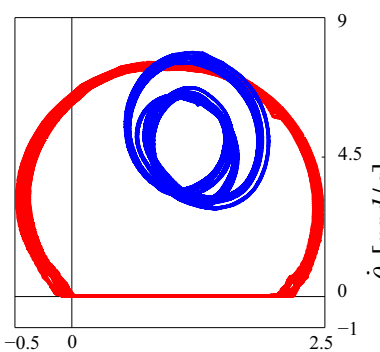

$\theta_{t}-\theta_{b}[\mathrm{rad}]$ (b)

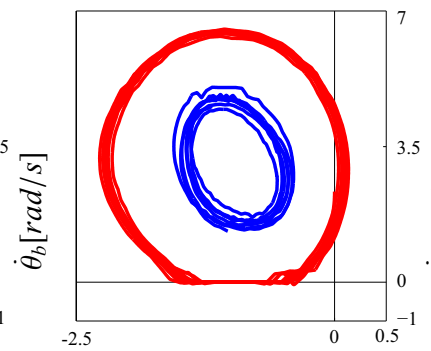

$\theta_{t}-\theta_{b}[\mathrm{rad}]$

(e)

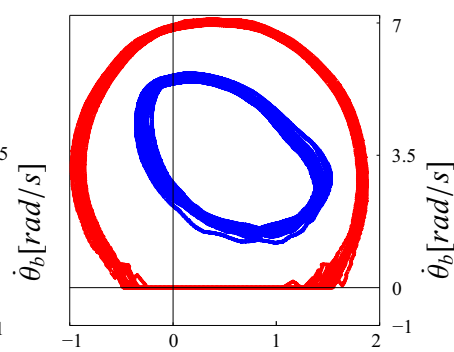

$\theta_{t}-\theta_{b}[\mathrm{rad}]$ (c)

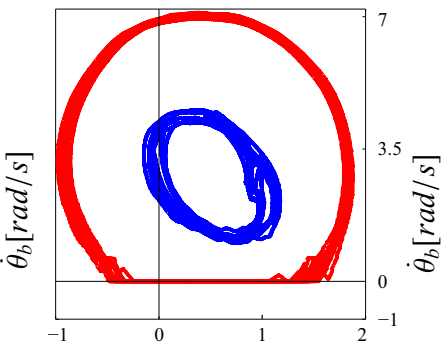

$\theta_{t}-\theta_{b}[\mathrm{rad}]$

(f)

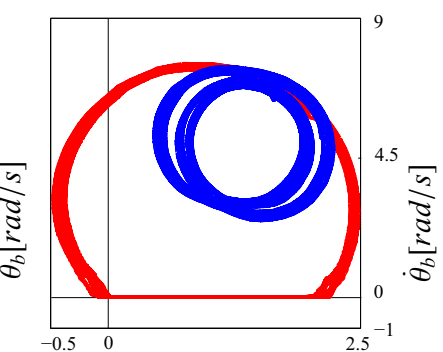

$\theta_{t}-\theta_{b}[\mathrm{rad}]$

Figure 3. Phase portraits of the drilling experiments using sliding-mode controller. The uncontrolled stick-slip trajectories and the controlled limit cycles are shown in red and blue respectively. The controller achieves (a) $47.86 \%$ (b) $59.26 \%$ (c) $51.52 \%$ (d) $57.58 \%$ (e) $66.72 \%$ (f) $64.72 \%$ reduction in vibration.

A response is considered of the stick-slip oscillations of the drill-bit, for WOB value of $W_{b}=1.76 \mathrm{kN}$, and a 1.5 inch pre-buckled flexible shaft and $T_{t}=39.57 \mathrm{Nm}$ torque generated by motor. Fig.2(a) presents its (top) experimental and (bottom) numerical time history. As can be seen, the top velocities in experiment and simulation (black curves) have sinusoidal vibrations, and the drill-bit velocities (red and green curves) experience stick-slip oscillations of almost constant amplitude. The numerical results were obtained using the model shown in Fig.2(b) with parameters identified as $k=10.00 \mathrm{Nm} / \mathrm{rad}$, $c=0.005 \mathrm{Nms} / \mathrm{rad}, J_{t}=13.93 \mathrm{~kg} \mathrm{~m}^{2}$ and $c_{t}=11.38 \mathrm{Nms} / \mathrm{rad}$. A TOB model is used for the TOB formulation, with corresponding parameters as developed in the previous work [4]. TOB recorded in the experiment and modelled can be seen in Fig.2(b) (blue curves). There is excellent agreement between the experimental observations and performed simulation.

After observing and modelling the stick-slip vibration, we focus on the suppression methods. To control stick-slip, one of the most commonly encountered vibration while drilling for oil and gas exploration, a sliding surface can be defined as

$s=\left(\dot{\theta}_{t}-\omega_{d}\right)+\lambda \int_{0}^{t}\left(\dot{\theta}_{t}-\omega_{d}\right) d \tau+\lambda \int_{0}^{t}\left(\dot{\theta}_{t}-\dot{\theta}_{b}\right) d \tau$, 
where $\theta_{t}$ and $\theta_{b}$ are angular positions of the rotary table and the drill-bit respectively, $\omega_{d}$ is the desired angular velocity and $\lambda$ is a positive control parameter. Based on the defined surface, then a new sliding-mode controller has been successfully designed and applied to lead the system to the sliding surface $(s=0)$, which leads the system to its equilibrium $\left(\dot{\theta}_{t}=\dot{\theta}_{t}=\omega_{d}\right)$ [5]. In presence of the delay in the motor, the controller has been tested in several different cases. Fig. 3 presents phase portraits of the drilling experiments using sliding-mode controller. The uncontrolled stick-slip trajectories and the controlled limit cycles are shown in red and blue respectively. The controller achieves (a) $47.86 \%$ (b) $59.26 \%$ (c) $51.52 \%$ (d) $57.58 \%$ (e) $66.72 \%$ (f) $64.72 \%$ reduction in vibration.

\section{Conclusion}

In this paper we have presented a lumped mass model for the drilling rig shown in Fig.1. By using identified model parameters in the preformed simulation, an excellent match between experiment and simulation is achieved capturing the stick-slip vibration. The next step of this study was to adapt a sliding-mode control method and applied to the obtained model in order to eliminate the stick-slip vibration observed in the drilling rig and simulation. The controller implemented in the experiment is successful in eliminating the stick-slip vibration in the presence of a delay and dead-zone in the actuator. Several experimental results were presented in this paper with a variety of the estimated parameters applied to the controller. A significant reduction in vibration amplitude is observed when the controller is applied.

Taking into consideration the positive experimental results reported in this paper, we can conclude that a robust mathematical model and a sliding-mode controller have been developed, which proved to predict accurately the responses of the analyzed experimental setup and eliminate the stick-slip oscillations and reduce it to torsional vibration in presence of delay in the actuator.

\section{References}

[1] M. Wiercigroch, Modelling and Analysis of BHA and Drill-string Vibrations R\&D Project sponsored by the BG Group (2010)

[2] P. Spanos, A. Chevallier, N. Politis, M. Payne, The Shock and Vibration Digest 35, 81 (2003)

[3] P.A. Patil, C. Teodoriu, Journal of Petroleum Science and Engineering 112, 227 (2013)

[4] M. Kapitaniak, V. Vaziri, J. Páez Chávez, K. Nandakumar, M. Wiercigroch, International Journal of Mechanical Sciences 101102, 324 (2015)

[5] V. Vaziri, Ph.D. thesis, University of Aberdeen (2015)

[6] M. Kapitaniak, Ph.D. thesis, University of Aberdeen (2015)

[7] M. Kapitaniak, V. Vaziri, J. Páez Chávez, M. Wiercigroch, Mechanical Systems and Signal Processing 100, 454 (2018)

[8] E. Navarro-López, R. Suárez, IEEE Conference on Control Applications pp. 14541460 (September 2004)

[9] E. Navarro-López, E. Licéaga-Castro, Chaos, Solitons and Fractals 41, 2035 (2009)

[10] Y. Liu, IET Control Theory \& Applications 9, 91 (2015)

[11] T. Vromen, C.H. Dai, N. Van De Wouw, T. Oomen, P. Astrid, H. Nijmeijer, IFAC Proceedings Volumes 48, 266 (2015) 